\title{
Existence of the Abrikosov vortex state in two-dimensional type-II superconductors without pinning.
}

\author{
A. V. Nikulov \\ Institute of Microelectronics Technology and High Purity Materials, Russian Academy of Sciences, 142432 Chernogolovka, \\ Moscow District, RUSSIA
}

\begin{abstract}
A nonperturbative fluctuation theory of the mixed state of two-dimensional type-II superconductor with finite size in the lowest Landau level approximation is proposed. The thermodynamic averages of the spatial average order parameter and of the Abrikosov parameter $\beta_{a}$ are calculated. It is shown that the thermodynamic fluctuations eliminate the Abrikosov vortex state in a wide region of the mixed state of two-dimensional type-II superconductor with real size.
\end{abstract}

PACS numbers: 74.60.Ge

Fluctuating behavior near second critical field, $H_{c 2}$, in type-II superconductors has recently been under intense experimental [1 5] and theoretical [6- 18] study, particularly in connection with high temperature superconductors (HTSC). Investigation of the fluctuations changes step by step our habitual notion about nature of the mixed state. Concepts "vortex lattice melting" and "vortex liquid" [6,7] have become very popular on first stage of a stormy investigation of fluctuation effects in HTSC. The vortex lattice melting transition is determined experimentally by a change of resistive properties in a perpendicular magnetic field [1, 3]. This transition was observed in bulk conventional superconductors before the HTSC discovery [19]. It was interpreted in 19 as a transition from the Abrikosov state into a one-dimensional state. But the vortex liquid as well as the one-dimensional state is not a new genuine thermodynamic state which is qualitatively different from the normal state [6]. Therefore the interpretations by [1],3] and by [19] are just the same. The vortex lattice melting is the transition from the Abrikosov state into the normal state with superconducting fluctuations (which was called as the one-dimensional state in 19, 18]. The experimental investigations show that this transition occurs below $H_{c 2}$ in all case [20,21, 1, 3]. The second critical field line $H_{c 2}(T)$ only marks a crossover from the normal metal to a strongly fluctuating superconducting state [6]. I think that the widespread term "vortex lattice melting" is not enough right. Therefore I will use the term "transition from the Abrikosov state into the normal state" in exchange for it.

The starting point of vortex lattice melting theories is assumption of the existence of the Abrikosov state at low temperatures. This assumption bases oneself on experimental evidence only. But real samples with finite sizes are investigated experimentally whereas the thermodynamic limit is used and a influence of disorder (pinning centers) is not considered in the theory. Therefore the experimental evidence may be not enough. The perturbation fluctuation theory [22, 14] shows no sign of the Abrikosov state. Moreover, according to [16] the fluctuation eliminates the Abrikosov state in superconductors with infinite sizes. The reduction of effective dimension- ality of the fluctuation in a magnetic field [23] and the destruction of off-diagonal long range order by thermal fluctuations in the mixed state [24, 9, 11, 17] cause doubt of the existence of the Abrikosov state in the thermodynamic limit also. According to results of 24] the fluctuation can not be considered as a perturbation in the mixed state of type-II superconductor with infinite sizes. Therefore a nonperturbative fluctuation theory of superconductors with finite sizes is required.

This theory for a most easy case is presented in this work. A two-dimensional superconductor in a perpendicular magnetic field near $H_{c 2}$ is considered. In this case the lowest Landau level (LLL) approximation is valid and the system is zero-dimensional 15. Therefore the exact thermodynamic averages can be calculated easily. The theory is based on a new expression of the free energy obtained from the Ginsburg-Landau (GL) one, $F_{G L}$. The results obtained in this work are most close to one obtained in [9]. In comparison with [9] a more easy method of calculation is suggested here and the concept of a transition into the Abrikosov state of two-dimensional superconductors with finite sizes is proposed.

We will proceed from the accurate expression for the free energy

$$
F=-k_{B} T \ln \sum \exp \left(-\frac{F_{G L}}{k_{B} T}\right)
$$

Strongly type-II superconductors near $H_{c 2}$ will be considered there. In this case one can neglect the fluctuations in the magnetic field. The problem of fluctuations can be investigated within a framework of the GL free energy functional, with the order parameter confined to the lowest Landau level (LLL) for Cooper pairs. In this approximation the GL free energy functional for two-dimensional superconductor may be write as (see [15])

$$
\frac{F_{G L}}{k_{B} T}=V\left(\varepsilon \mid \overline{|\Psi|^{2}}+0.5 \beta_{a}{\overline{|\Psi|^{2}}}^{2}\right)
$$

$\varepsilon=G i^{-1 / 2}(h t)^{-1 / 2}\left(h-h_{c 2}\right), G i=\left[2 k_{B} T / H_{c}^{2}(0) d \xi^{2}(0)\right]$ is the Ginsburg number in zero magnetic field; $t=T / T_{c}$; $h=H / H_{c 2}(0) ; H_{c 2}(0)=-T_{c}\left(d H_{c 2} / d T\right)_{T=T_{c}} ; H_{c}(0)=$ $-T_{c}\left(d H_{c} / d T\right) ; H_{c}$ is the thermodynamic critical field; 
$\xi(0)$ is the coherence length at $\mathrm{T}=0 . \mathrm{d}$ is the film thickness; $\mathrm{V}$ is the film area; $\overline{|\Psi|^{2}}=\left(\int_{V} d^{2} r|\Psi|^{2}\right) / V$ is the spa-

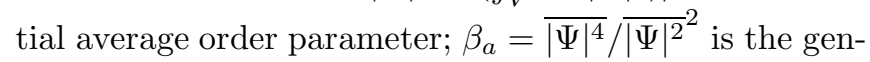
eralized Abrikosov parameter; $\overline{|\Psi|^{4}}=\left(\int_{V} d^{2} r|\Psi|^{4}\right) / V$. We use a dimensionless unit system in which $l_{x}=1$; $l_{y} / 2=1 ; l_{x} l_{y} / 2=\Phi_{0} / H=1 ; \Psi=\left(d \beta l_{x} l_{y} / 2 k_{B} T\right)^{1 / 4} \psi$. In this unit system the film area $\mathrm{V}$ is equal the degeneracy number of the LLL. $l_{x}, l_{y}$ are the parameter of the triangular Abrikosov lattice corresponding to a magnetic field value $H ; \Phi_{0}$ is the flux quantum; $\psi$ is order parameter in the conventional units. To obtain (2) we used the well-known relation: $\alpha=-e \hbar H_{c 2} / m c ; \alpha^{2} / 2 \beta=H_{c}^{2} / 8 \pi$. $\alpha$ and $\beta$ are the conventional coefficients of GL free energy 25 .

In the LLL approximation the sum in (1) is to be taken over the subspace spanned by the LLL. To substitute the sum in (1) on two integral

$F=-k_{B} T \ln \left[\int_{0}^{\infty} d \overline{|\Psi|^{2}} \int_{\beta_{A}}^{\infty} d \beta_{a} N\left(\overline{|\Psi|^{2}} ; \beta_{a}\right) \exp \left(-\frac{F_{G L}}{\left.k_{B} T\right)}\right]\right.$

$N\left(\overline{|\Psi|^{2}} ; \beta_{a}\right) d \overline{|\Psi|^{2}} d \beta_{a}$ is a subspace volume with the given values of $\overline{|\Psi|^{2}}$ and $\beta_{a} . \beta_{A}$ is the minimum value of $\beta_{a}$ in the subspace spanned by LLL. It was shown in 26 that $\beta_{A}$ correspond to the triangular lattice and is equal approximately 1.159595 .

To expand the order parameter on the Landau eigenfunctions $\Psi(r)=V^{-1 / 2} \sum_{\lambda} \Psi_{\lambda} \varphi_{\lambda}(r)$. Then $\overline{|\Psi|^{2}}=$ $V^{-1} \sum_{\lambda}\left|\Psi_{\lambda}\right|^{2}=V^{-1} \sum_{\lambda}\left[\left(R e \Psi_{\lambda}\right)^{2}+\left(\operatorname{Im} \Psi_{\lambda}\right)^{2}\right] . \quad \mathrm{A}$ eigenfunction number is equal the degeneracy number of the LLL, which is equal in our unit system $\mathrm{V}$. Consequently the given values of $\overline{|\Psi|^{2}}$ lie on a $2 \mathrm{~V}$ dimensional sphere with radius ${\overline{|\Psi|^{2}}}^{0.5}:\left({\overline{|\Psi|^{2}}}^{0.5}\right)^{2}=$ $\sum_{\lambda}\left[\left(\operatorname{Re} \Psi_{\lambda} / V^{0.5}\right)^{2}+\left(\operatorname{Im} \Psi_{\lambda} / V^{0.5}\right)^{2}\right]$. The area of this sphere is equal $2 \pi^{V} V !\left({\overline{|\Psi|^{2}}}^{0.5}\right)^{2 V-1}$. It is obvious that $\beta_{a}$ value does not depend on the $\overline{|\Psi|^{2}}$ value. Therefore $N\left(\overline{|\Psi|^{2}} ; \beta_{a}\right)$ is proportional to ${\overline{|\Psi|^{2}}}^{V-0.5} n\left(\beta_{a}\right) . n\left(\beta_{a}\right)$ is a fraction of the $2 \mathrm{~V}$-dimensional sphere with the given value of $\beta_{a}$. Multipliers independent of $\overline{|\Psi|^{2}}$ and $\beta_{a}$ are omitted.

To write the generalized Abrikosov parameter through eigenvalues

$\beta_{a}=\left(\sum_{\lambda_{i}} V_{\lambda_{1}, \lambda_{2}, \lambda_{3}, \lambda_{4}} \Psi_{\lambda_{1}}^{*} \Psi_{\lambda_{2}}^{*} \Psi_{\lambda_{3}} \Psi_{\lambda_{4}}\right) /\left(\sum_{\lambda}\left|\Psi_{\lambda}\right|^{2}\right)^{2}$. $V_{\lambda_{1}, \lambda_{2}, \lambda_{3}, \lambda_{4}}=V^{-1} \int_{V} d^{2} r \varphi_{\lambda_{1}}^{*} \varphi_{\lambda_{2}}^{*} \varphi_{\lambda_{3}} \varphi_{\lambda_{4}}$. We shall use the Eilenberger basis function [27]. In this basis each $\varphi_{\lambda}$ function describes the triangular Abrikosov lattice and $\lambda=\left(\lambda_{x} ; \lambda_{y}\right)$ is their relative displacement. $\lambda_{x}=$ $\left(l_{x}^{2} / L_{y}\right) n ; \lambda_{y}=\left(l_{y}^{2} / L_{x}\right) m . \mathrm{n} ; \mathrm{m}$ are integer numbers; $L_{x} ; L_{y}$ are film sizes. $L_{x} L_{y}=V . V_{\lambda_{1}, \lambda_{2}, \lambda_{3}, \lambda_{4}}$ was calculated in 28.

Let $\Psi_{\lambda=0}=\Psi_{0}=1$. Following [24] I introduce new variables $u_{+}(\lambda)$ and $u_{-}(\lambda)$

$$
\Psi_{\lambda}=\left(\left|V_{0,0, \lambda,-\lambda}\right| / 2 V_{0,0, \lambda,-\lambda}\right)^{1 / 2}\left[u_{+}(\lambda)+u_{-}(\lambda)\right]
$$

$$
\Psi_{-\lambda}=\left(\left|V_{0,0, \lambda,-\lambda}\right| / 2 V_{0,0, \lambda,-\lambda}\right)^{1 / 2}\left[u_{+}^{*}(\lambda)-u_{-}^{*}(\lambda)\right]
$$

Then the generalized Abrikosov parameter may be write as

$$
\begin{gathered}
\beta_{a}=\beta_{A}+\frac{\sum_{\lambda \neq 0}\left(\Gamma_{+}\left|u_{+}\right|^{2}+\Gamma_{-}\left|u_{-}\right|^{2}\right)+U-B}{\left[1+0.5 \sum_{\lambda \neq 0}\left(\left|u_{+}\right|^{2}+\left|u_{-}\right|^{2}\right)\right]^{2}} \\
\Gamma_{+}=2 V_{0, \lambda, 0, \lambda}+\left|V_{0,0, \lambda,-\lambda}\right|-\beta_{A} \\
\Gamma_{-}=2 V_{0, \lambda, 0, \lambda}-\left|V_{0,0, \lambda,-\lambda}\right|-\beta_{A}
\end{gathered}
$$

$$
\begin{gathered}
U=\sum_{\lambda_{i} \neq 0} V_{\lambda_{1}, \lambda_{2}, \lambda_{3}, \lambda_{4}} \Psi_{\lambda_{1}}^{*} \Psi_{\lambda_{2}}^{*} \Psi_{\lambda_{3}} \Psi_{\lambda_{4}} \\
B=\beta_{A}\left(\sum_{\lambda \neq 0}\left|\Psi_{\lambda}\right|^{2}\right)^{2}
\end{gathered}
$$

The sum by all $\lambda \neq 0$.

At $B \ll 1, \beta_{a} \simeq \beta_{A}+\sum_{\lambda \neq 0}\left(\Gamma_{+}\left|u_{+}\right|^{2}+\Gamma_{-}\left|u_{-}\right|^{2}\right)$. Consequently at $\beta_{a}-\beta_{A} \ll 1$ the given value $\beta_{a}$ lie in a $2 \mathrm{~V}$-dimensional circle. A "length" of this circle is proportional to $\left[\left(\beta_{a}-\beta_{A}\right)^{0.5}\right]^{2 V-2}$. Therefore $n\left(\beta_{a}\right)$ is proportional to $\left(\beta_{a}-\beta_{A}\right)^{V-1}$ at $\beta_{a}-\beta_{A} \ll 1$. At a larger $\beta_{a}-\beta_{A}$ value $n\left(\beta_{a}\right)$ increases more slowly with the $\beta_{a}-\beta_{A}$ increasing. In this case one may introduce a new function and write $n\left(\beta_{a}\right)=\left[f\left(\beta_{a}-\beta_{A}\right)\right]^{V-1}$. Taking into account that $V \gg 1$ we may overwrite (1a) as

$$
F=-k_{B} T \ln \left[\int_{0}^{\infty} d \overline{|\Psi|^{2}} \int_{\beta_{A}}^{\infty} d \beta_{a} \exp \left(-\frac{V F_{N e w}}{k_{B} T}\right)\right]
$$

where $F_{N e w}$ is the new free energy expression in which the entropy term connected with $\overline{|\Psi|^{2}}$ and $\beta_{a}$ values is take into account

$$
\frac{F_{N e w}}{k_{B} T}=\varepsilon \mid \overline{\left.\Psi\right|^{2}}+0.5 \beta_{a}{\overline{|\Psi|^{2}}}^{2}-\ln \overline{|\Psi|^{2}}-\ln \left[f\left(\beta_{a}-\beta_{A}\right)\right]
$$

Because $V \gg 1$ the exact thermodynamic averages of the $\overline{|\Psi|^{2}}$ and $\beta_{a}$ values, $<\overline{|\Psi|^{2}}>$ and $\left\langle\beta_{a}>\right.$, are close to values corresponded to the $F_{N e w}$ minimum. Consequently these values are specified by relations

$$
\begin{aligned}
<\overline{|\Psi|^{2}}>= & {\left[\left(\frac{\varepsilon}{2<\beta_{a}>}\right)^{2}+\frac{1}{<\beta_{a}>}\right]^{1 / 2}-\frac{\varepsilon}{2<\beta_{a}>} } \\
& \frac{f^{\prime}\left(<\beta_{a}>-\beta_{A}\right)}{f\left(<\beta_{a}>-\beta_{A}\right)}=0.5<\overline{|\Psi|^{2}}>^{2}
\end{aligned}
$$


The (5a) relation coincide with one obtained in 16] and is close to the entropy dependence obtained by O'Neill and Moore [9]. This relation is close to the Abrikosov solution, $\left\langle\overline{|\Psi|^{2}}\right\rangle=-\varepsilon \mid\left\langle\beta_{a}\right\rangle$, at $-\varepsilon \gg 1$ (below the $H_{c 2}$ critical region) and to the result of the fluctuation theory in the linear approximation, $<\overline{|\Psi|^{2}}>=1 / \varepsilon$, at $\varepsilon \gg 1$ (above the $H_{c 2}$ critical region).

The $f\left(\beta_{a}-\beta_{A}\right)$ function is not evaluated completely in this work. Therefore we calculate the thermodynamic averages of $\beta_{a}$ below the critical region only. At $-\varepsilon \gg 1$, $<\beta_{a}>-\beta_{A} \ll 1$. Consequently $f\left(<\beta_{a}>-\beta_{A}\right) \simeq<$ $\beta_{a}>-\beta_{A}$ and according to (5b)

$$
\begin{gathered}
<\beta_{a}>-\beta_{A}=2<\overline{|\Psi|^{2}}>^{-2} \simeq 2\left(\frac{\beta_{A}}{\varepsilon}\right)^{2}= \\
2 \beta_{A}^{2} \operatorname{Giht}\left(h_{c 2}-h\right)^{-2}
\end{gathered}
$$

The Ginsburg number values of conventional twodimensional type-II superconductors lie in the interval $3 * 10^{-6}<G i<3 * 10^{-3}$. Therefore in the center of the mixed state region, at $\mathrm{t}=0.5, \mathrm{~h}=0.25$, $10^{-5}<\left(<\beta_{a}>-\beta_{A}\right)<10^{-2}$.

According (1b) there is not thermodynamic singularity in two-dimensional type-II superconductor which may be connected with a phase transition from normal state into Abrikosov vortex state. Therefore one should think that this transition occurs without thermodynamic singularity. I propose there a concept of this transition based on the difference between the Abrikosov state and the normal state with superconducting fluctuation in the Eilenberger function basis [27]. It is obvious that above the $H_{c 2}$ critical region all thermodynamic average of the Eilenberger eigenvalue squares, $<\left|\Psi_{\lambda}\right|^{2}>$, are equal and $<|\Psi(r)|^{2}>$ is constant in space. Consequently, in the normal state with superconducting fluctuation (in the vortex liquid phase [6]) $<\overline{|\Psi|^{2}}>=V^{-1} \sum_{\lambda}<\left|\Psi_{\lambda}\right|^{2}>=<\left|\Psi_{\lambda=0}\right|^{2}>$ whereas in the Abrikosov state $<\overline{|\Psi|^{2}}>=V^{-1}<\left|\Psi_{\lambda=0}\right|^{2}>$ in the mean field approximation. Therefore one should think that the transition into the Abrikosov vortex state take place when a one Eilenberger eigenvalue, $\Psi_{0}$, brings the main contribution to the order parameter: $\left\langle\left|\psi_{0}\right|^{2}\right\rangle=<$ $\left|\Psi_{0}\right|^{2}>/ V<\overline{|\Psi|^{2}}>=\left(1+\sum_{\lambda \neq 0}<\left|\psi_{\lambda}\right|^{2}>\right)^{-1}=$ $\left(1+0.5 \sum_{\lambda \neq 0}<\left|u_{+}\right|^{2}>+<\left|u_{-}\right|^{2}>\right)^{-1} \simeq 1$. Where $<\left|\psi_{\lambda}\right|^{2}>=<\left|\Psi_{\lambda}\right|^{2}>/<\left|\Psi_{0}\right|^{2}>$.

The $\left\langle\left|\psi_{0}\right|^{2}>\right.$ value is connected with $\left\langle\beta_{a}\right\rangle-\beta_{A}$ value. We shall consider the region below the $H_{c 2}$ critical region where $\left\langle\beta_{a}>-\beta_{A} \ll 1\right.$. Following to [24] one may show that $\Gamma_{+} \simeq 2 \beta_{A}$ and $\Gamma_{-} \simeq 3.1|\lambda|^{4}$ at $|\lambda| \ll 1$. Consequently, according (3a), $\sum_{\lambda \neq 0}\left|u_{+}\right|^{2} \ll 1$ and it may be that $\left|u_{-}\right|^{2} \gg 1$ at $\beta_{a}-\beta_{A} \ll 1$. Therefore we will consider states with $u_{+}=0$ only. In this case $\left|\psi_{0}\right|^{2}=\left(1+0.5 \sum_{\lambda \neq 0}\left|u_{-}\right|^{2}\right)^{-1}$ and

$$
\beta_{a}-\beta_{A}=\frac{\sum_{\lambda \neq 0} \Gamma_{-}\left|u_{-}\right|^{2}+U-B}{\left(1+0.5 \sum_{\lambda \neq 0}\left|u_{-}\right|^{2}\right)^{2}}
$$

$U=0.25 \sum_{\lambda_{i} \neq 0} V_{\lambda_{1}, \lambda_{2}, \lambda_{3}, \lambda_{4}} \exp \left[0.5 i\left(\varphi_{\lambda_{1}}+\varphi_{\lambda_{2}}-\varphi_{\lambda_{3}}-\right.\right.$ $\left.\left.\varphi_{\lambda_{4}}\right)\right] u_{-}^{*}\left(\lambda_{1}\right) u_{-}^{*}\left(\lambda_{2}\right) u_{-}\left(\lambda_{3}\right) u_{-}\left(\lambda_{4}\right)$;

$B=\beta_{A}\left(0.5 \sum_{\lambda \neq 0}\left|u_{-}\right|^{2}\right)^{2}$. Where $\exp \left(-i \varphi_{\lambda}\right)=$ $\left|V_{0,0, \lambda,-\lambda}\right| / V_{0,0, \lambda,-\lambda}$.

At $0.5 \sum_{\lambda \neq 0}\left|u_{-}\right|^{2} \ll 1$ we have the Abrikosov state with fluctuation and at $0.5 \sum_{\lambda \neq 0}\left|u_{-}\right|^{2} \gg 1$ we have the normal state with superconducting fluctuations. All states corresponding to very small $\beta_{a}-\beta_{A}$ value lie near the Abrikosov state $\left(0.5 \sum_{\lambda \neq 0}\left|u_{-}\right|^{2} \ll 1\right)$. At larger $\beta_{a}-\beta_{A}$ value states exist which are removed from the Abrikosov state (states with $0.5 \sum_{\lambda \neq 0}\left|u_{-}\right|^{2} \gg 1$ ). The critical value, $\left(\beta_{a}-\beta_{A}\right)_{c}$, exists below which states with $0.5 \sum_{\lambda \neq 0}\left|u_{-}\right|^{2} \gg 1$ appear. I suppose that transition into Abrikosov state occurs when $\left\langle\beta_{a}\right\rangle-\beta_{A}=$ $\left(\beta_{a}-\beta_{A}\right)_{c}$.

The $\left(\beta_{a}-\beta_{A}\right)_{c}$ value is not calculated in this paper. I will show only that in two-dimensional superconductor states with $0.5 \sum_{\lambda \neq 0}\left|u_{-}\right|^{2} \gg 1$ exist at small $\beta_{a}-\beta_{A}$ value.

We will consider the states with $u_{-}\left(\lambda_{x}=0 ; \lambda_{y}\right)=$ $u /\left(\lambda_{y} L_{x}\right)=u / m$ at $\left|\lambda_{y}\right| \leq l \leq 0.1, \mathrm{~m}$ is odd number and $u_{-}\left(\lambda_{x} ; \lambda_{y}\right)=0$ at all other $\lambda_{x}, \lambda_{y}$ values. In this case

$$
\beta_{a}-\beta_{A}=\left(\frac{0.52 l^{3} u^{2}+(0.87 / l-2.8 l) u^{4}}{\left(1+1.23 u^{2}\right)^{2}}\right) \frac{1}{L_{x}}
$$

At $0.5 \sum_{\lambda \neq 0}\left|u_{-}\right|^{2}=1.23 u^{2} \gg 1, \beta_{a}-\beta_{A} \simeq(0.58 / l-$ $1.86 l) / L_{x}$. The relation (3c) is valid at $l \leq 0.1$ only. At $\mathrm{l}=0.1, \beta_{a}-\beta_{A}=5.6 / L_{x}$. Consequently $\left(\beta_{a}-\beta_{A}\right)_{c} \leq$ $5.6 / L_{x}$.

Thus the obtained results show that the Abrikosov vortex state can not exist in two-dimensional type-II superconductors with infinite sizes (at $L_{x}=\infty,\left(\beta_{a}-\beta_{A}\right)_{c}=0$ and consequently $\left\langle\beta_{a}\right\rangle-\beta_{A}>\left(\beta_{a}-\beta_{A}\right)_{c}$ always). The same result was obtained in [9, [16].

In "dirty" $\left(G i \simeq 310^{-3}\right)$ thin films with usual sizes $\left(L_{x}=L_{x} / l_{x}=10^{3}-10^{5}\right)$ the transition into Abrikosov state takes place much below $H_{c 2}$ (for example at $\mathrm{t}=$ 0.5 and $G i=3 * 10^{-3},<\beta_{a}>-\beta_{A}<\left(\beta_{a}-\beta_{A}\right)_{c} \leq$ $5.6 / L_{x}=5 * 10^{-3}-5 * 10^{-5}$ below $h=0.25 \simeq 0.5 h_{c 2}$ only, see above). It must be emphasize that these conclusion are valid for samples without pinning centers only. Any pinning of the flux lines by disorder, ets., will inhibit and slow the fluctuations and will stabilize the Abrikosov state [9].

According to the concept described above, the Abrikosov state and the normal state with superconducting fluctuation have different distributions of the order parameter in the space. Therefore a transition from the Abrikosov state into the normal state (vortex lattice melting) can be observed experimentally by pinning 
appearance (by a change of resistive properties in a perpendicular magnetic field). It was observed in [1,5] that this transition occurs (pinning appears) much below $H_{c 2}$ in thin amorphous films with a small amount of pinning centers. It may be considered an experimental confirmation of the result obtained above.

I would like to thank Z.Tesanovic' for sending his paper. The results of these papers stimulated this work. This work was supported by the National Scientific Council on High Temperature Superconductivity, Project 93195 .

1 P.Berghuis and P.H.Kes, Phys.Rev. B 47, 262 (1993); P.Koorevaar, P.H.Kes, A.E.Koshelev, and Aarts, Phys.Rev.Lett. 72, 3250 (1994); P.Berghuis, A.L.F. van der Slot, and P.H.Kes, Phys.Rev.Lett. 65, 2683 (1990).

2 A.Yazdani, W.R.White, M.R.Hahn, M.Gabay, M.R.Beasley, and A.Kapitulnik, Phys.Rev.Lett. 70, 505 (1993).

3 W.K.Kwok, J.Fendrich, S.Flesher, U.Welp, J.Downey, and G.W.Crabtree, Phys.Rev.Lett. 72, 1092 (1994).

4 V.A.Marchenko and A.V.Nikulov, Physica C 210, 466, (1993).

5 A.V.Nikulov, D.Yu.Remisov and V.A.Oboznov, Physica C 235-240, 1945 (1994); A.V.Nikulov, D.Yu.Remisov and V.A.Oboznov, submitted to Phys.Rev.Lett.

6 G.Blatter, M.V.Feigel'man, V.B.Geshkenbein, A.I.Larkin, and V.M.Vinokur, Rev.Mod.Phys. 66, 1130 (1994)

7 D.S.Fisher, M.P.A.Fisher, and D.A.Huse, Phys.Rev. B 43, 130 (1991).

8 K.Maki and R.S.Thompson, Physica C 162-164, 275, (1989).

9 M.A.Moore, Phys.Rev. B 39, 136 (1989); M.A.Moore, Phys.Rev. B 45, 7336 (1992); N.K.Wilkin and M.A.Moore, Phys.Rev. B 47, 957, (1993); J.A.O'Neill and M.A.Moore, Phys.Rev. B 48, 374, (1993); J.A.O'Neill and M.A.Moore, Phys.Rev.Lett. 69, 2582, (1992).

10 H.H.Lee and M.A.Moore, Phys.Rev. B 49, 9240 (1994).

11 R.Ikeda, T.Ohmi, and T.Tsuneto, J.of Phys.Society of Japan 59, 1740 (1990); R.Ikeda, T.Ohmi, and T.Tsuneto, J.of Phys.Society of Japan 61, 254 (1992).

12 A.Houghton, R.A.Pelcovits, and A.Sudbo, Phys.Rev. B 42, 906 (1990)

13 Shinobu Hikami and Ayumi Fujita, Phys.Rev. B 41, 6379 (1990).

14 E.Brezin, A.Fujita, and S.Hikami, Phys.Rev.Lett. 65, 1949, (1990); S.Hikami, A.Fujita, and A.I.Larkin, Phys.Rev. B 44, 10400 (1991).

15 Zlatko Tesanovic' and Lei Xing, Phys.Rev.Lett. 67, 2729, (1991); Zlatko Tesanovic', Phys.Rev. B 44, 12635 (1991); Zlatko Tesanovic', Lei Xing, Lev Bulaevskii, Qiang Li, and M.Suenaga, Phys.Rev.Lett. 69, 3563 (1992); Zlatko Tesanovic' and A.V.Andreev, Phys.Rev. B 49, 4064
(1994)

16 Zlatko Tesanovic', Physica C 220, 303, (1994).

17 G.G.Sergeeva, Fiz.Nizk.Temp. 20, 3 (1994).

18 A.V.Nikulov, Supercond.Sci.Technol. 3, 377 (1990).

19 V.A.Marchenko and A.V.Nikulov, Pisma Zh.Eksp.Teor.Fiz. 34, 19 (1981) (JETP Lett. 34, 17 (1981)).

20 V.A.Marchenko and A.V.Nikulov, Zh.Eksp.Teor.Fiz. 80, 745 (1981) (Sov.Phys.-JETP 53, 377 (1981)).

21 V.A.Marchenko and A.V.Nikulov, Zh.Eksp.Teor.Fiz. 86, 1395 (1984) (Sov.Phys.-JETP 59, 815 (1984)).

22 G.J.Ruggeri and D.J.Thouless, J.Phys. F 6, 2063 (1976)

23 P.A.Lee and S.R.Shenoy, Phys.Rev.Lett. 28, 1025 (1972).

24 K.Maki and H.Takayama, Progr.Theor.Phys. 46, 1651 (1971).

25 P.G. De Gennes, Superconductivity of Metals and Alloys (1966).

26 W.H.Kleiner, L.M.Roth and S.H.Autler, Phys.Rev. 133A, 1226 (1964)

27 G.Eilenberger, Phys.Rev. 164, 628 (1967).

28 G.J.Ruggeri, Phys.Rev. B 20, 3626 (1979). 